\title{
Successful Management of Recurrent Neuroendocrine Carcinoma of the Sphenoid Sinus Using Octreotide LAR: A Case Report
}

Liuyiqian, Caixiaomin, Zhuweiyou, Zhenfuxi* and Guyanhong*

The First Affiliated Hospital with Nanjing Medical University, Nanjing 210029, PR China

"Corresponding author: Guyanhong, Department of Oncology, The First Affiliated Hospital with Nanjing Medical University, Nanjing 210029, PR China, Tel: +8613813908678; E-mail: guluer@163.com Zhenfuxi, Department of Thoracic surgery, The First Affiliated Hospital with Nanjing Medical University, Nanjing 210029, PR China, Tel:+8613770606757; E-mail: zfxnjmu@sina.com

Received date: March 10, 2017; Accepted date: March 24, 2017; Published date: March 29, 2017

Copyright: ( 2017 Xiaomin C, et al. This is an open-access article distributed under the terms of the Creative Commons Attribution License, which permits unrestricted use, distribution, and reproduction in any medium, provided the original author and source are credited.

\begin{abstract}
Overexpression of somatostatin (SST) receptors detected in most neuroendocrine carcinomas (NECs) appears to play an important role in the pathogenesis of NECs. Inhibition of SST receptors with SST analogues has been proved to be effective in various types of neuroendocrine tumors. We present here a case report of a 59-year-old female with recurrent sphenoidal NEC treated with SST analogues Octreotide and Octreotide long-acting release (LAR) as a salvage therapy. Improvement of optic nerve compression and quality of life (QOL) were observed along with a 77 months of progression free survival. This result suggests that OCT/OCT LAR may be a new approach to treating advanced NECs of the paranasal sinuses.
\end{abstract}

Keywords: Neuroendocrine carcinomas; Sphenoid sinus; Somatostatin analogues; Octreotide long-acting release

\section{Introduction}

Neuroendocrine carcinomas (NECs) are extremely rare in the sphenoid sinus and few treatment methods are currently available for this type of tumors. Located at the critical region contiguous with the central nervous system, sphenoidal carcinomas pose a particular challenge to complete resection of the tumor [1]. Moreover, the use of radiotherapy and/or chemotherapy is controversial as they have little effect on survival of patients in advanced disease [2].

SST is a ubiquitous peptide found throughout the body with various biological functions. It serves as a neurotransmitter, and inhibits both exocrine and endocrine secretions of the gastroenteropancreatic systems [3]. Previous studies have demonstrated that NEC cells express SST receptors (SSTRs) belonging to the G protein family $[4,5]$. The biological actions of SST are mediated through five subtypes of receptors (SSTRs 1-5). SST and its analogues have a potential inhibitory effect on the proliferation of neuroendocrine malignancies in various cell types. This effect may be attributed to the SST-induced elevation of activity of a tyrosine phosphatase SHP-1, resulting in cellcycle arrest/apoptosis of the tumor cells [6]. Here, we present a case report of successful use of SST analogue Octreotide (OCT) in a sphenoidal NEC patient whose disease had progressed despite previous combination treatment of surgery, radiotherapy and chemotherapy.

\section{Case Report}

In October 2008, a 59-year-old woman was admitted to our hospital because of transient syncope. A left side visual field defect was noted on physical examination and computed tomography (CT) scan demonstrated a mass in the sphenoid sinus. A nasal endoscopic sphenoid sinus surgery plus middle turbinectomy was performed for the mass. Histologic examination of the resected specimens revealed small to moderate size of cells containing eosinophilic cytoplasm, dispersed chromatin and inconspicuous nucleoli arranged in trabeculae or rosettes (Figure 1A-D). Immunohistochemical (IHC) examination of the tumor cells showed a positive reaction to synaptophysin and all subtypes of somatostatin receptors (SSTRs1-5) (Figure 1B), but a negative reaction to epithelial membrane antigen (EMA), cytokeratin and S-100 protein (Figures 1C and 1A). After surgery, the patient underwent local radiotherapy (a total of $66 \mathrm{~Gy}$ fractionated into 33 sessions) and four cycles of chemotherapy (cisplatin+ etoposide+gemcitabine).

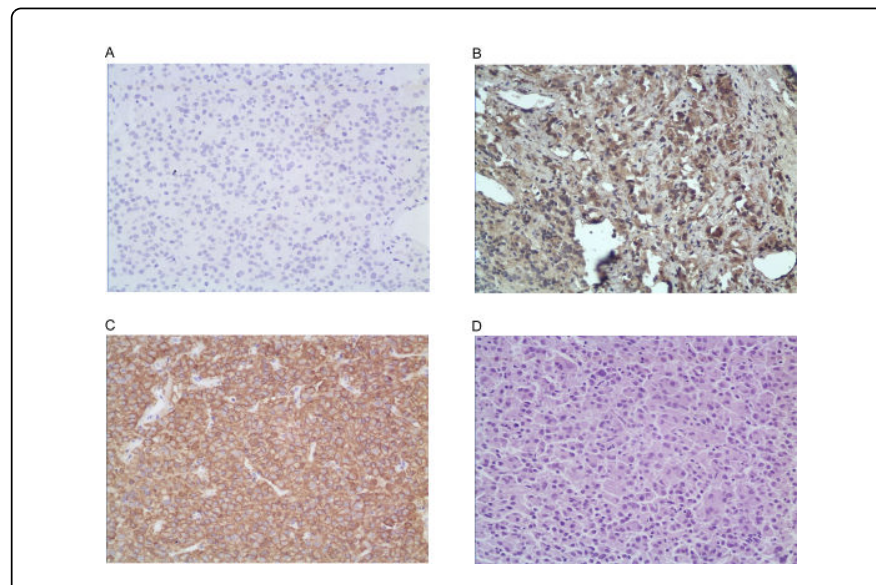

Figure 1: Histopathological and immunohistochemical (IHC) staining of the tumor $(\mathrm{x} 200)$. A: IHC staining showed a negative immunoreaction to EMA, cytokeratin and S-100 protein. B: IHC 1 staining of SSTRs 1-5 showed moderate to strong positive reaction in the cytoplasm. C: IHC staining showed a positive immunoreaction to synaptophysin. D: H\&E staining showed small to moderate size of cells containing eosinophilic cytoplasm, dispersed chromatin and inconspicuous nucleoli. 
Unfortunately, the patient experienced disease progression despite all these aforementioned therapies: The left visual field defect was extending and the patient complained of severe fatigue with persisting neutrophilic granulocytopenia during radiochemotherapy. Magnetic resonance imaging (MRI) revealed a protrusion into the posterior ethmoid sinus, with bilateral envelopment of the cavernous sinus, and invasion of the ethmoid bone involving right optic canal, indicating recurrence of the tumor (Figures $2 \mathrm{~A}$ and $2 \mathrm{~B}$ ). The quality of life was poor with a declining QOL value evaluated by EuroQoL-5D (EQ5D) (Figure 3).

The patient then agreed to try SST analogue OCT and OCT LAR. OCT was intravenously infused at an initial dose of $0.6 \mathrm{mg} / \mathrm{d}$ for 14 days followed by OCT LAR $20 \mathrm{mg}$ once a month.

Two months after OCT/OCT LAR treatment, symptoms of optic nerve compression were alleviated with a significant reduction both in tumor size and enhancement of tumor on contrasted MRI (Figures 2C and 2D). Up until May 2013, the patient well responded to OCT/OCT LAR treatment (Figures 2E-H), and remained clinically well with increased EQ-5D scores (Figure 3).
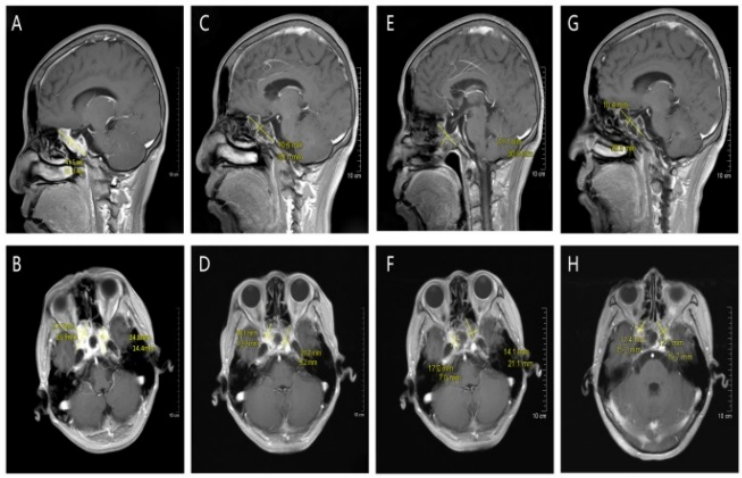

Figure 2: T1-weighted MRI images. Transverse (A) and sagittal (B) images before OCT treatment and after intravenous administration of Gd(III)-diethylenetriamine penta-acetic acid (Gd-DTPA): the tumor protruded into the posterior ethmoid sinus, enveloped the cavernous sinus bilaterally, invaded the ethmoid bone and involved the right side optic canal. Transverse (C) and sagittal (D) images showed a reduction in both tumor volume and tumor enhancement two months after OCT LAR therapy. E, F and G, H showed a stable disease status of the tumor 12 months and 36 months after application of OCT LAR, respectively.

OCT and OCT LAR were well tolerated, and the patient had no other adverse reactions than mild local reactions at the injection site. There was no significant change in either peripheral blood-cell counts or biochemical markers before and after OCT/OCT LAR therapy. The definition of drug-related adverse reactions was strictly according to the National Cancer Institute Common Toxicity Criteria (NCI-CTC) version 3.0.

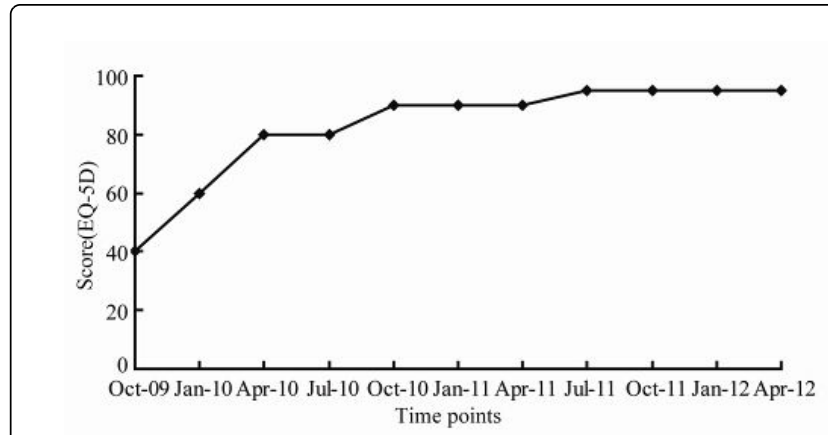

Figure 3: Quality of life evaluated by the EuroQoL-5D (EQ-5D). The patient had a low score of EQ-5D during radio-chemotherapy. After the administration of OCT, the score increased markedly and maintained at a high level for a long time.

\section{Discussion}

Until now, surgical resection remains the only curative therapy for NECs of the sphenoid sinuses. However, the sphenoid sinuses are sited at the central part of the skull base and adjacent to several important neurovascular structures. Therefore, an R0 resection for local advanced carcinoma of the sphenoid sinuses is rather a challenge for the surgeon [1]. In our case, only an R2 resection could be done as the tumor cells had invaded the optic nerve. Worse than that, the patient failed to respond to adjuvant radiotherapy 66 Gyand 4 cycles of combination chemotherapy cisplatin+etoposide+gemcitabine). Therefore, a new therapeutical strategy for advanced NEC of the sphenoid sinus is in urgent need for this kind of patients.

Expression of SSTRs is found in $80 \%-100 \%$ of neuroendocrine carcinomas [7]. Sphenoidal neuroendocrine carcinoma has to be differentiated from other neoplasms involving nasal cavity and paranasal sinuses such as squamous cell carcinoma, lymphoma, melanoma, olfactory neuroblastoma, and sinonasal undifferentiated carcinoma. In our present case, synaptophysin and SSTR1-5 were positive while EMAcytokeratin and S-100 were negative which favoured the diagnosis of neuroendocrine carcinoma over other neoplasms.Ligation of the SSTR by SST or SST analogues results in cellular internalization of the complex [8]. In vitro studies have shown that OCT inhibits tumor cell growth, stimulates apoptosis, induce indirect antiproliferative effects through anti-angiogenic mechanisms and plays an immunomodulatory role in the disease process of NECs [9]. It is also effective in suppressing excessive hormone secretion of functioning NECs. Thus, SST analogues have been widely used in treating gastroenteropancreatic neuroendocrine tumors uniformly expressing SSTRs.

OCT has a short half-life and is inconvenient to use: It has to be subcutaneously injected several times a day or to be intravenously infused. Stable compound such as OCT LAR is now available for a convenient monthly injection and improves patient compliance. Prolonged drug release of OCT LAR is achieved by encapsulation in microspheres composed of a tumor [10]. Fluctuation of blood drug level may be seen in the initial 7-14 days. After prolonged use of the agent, however, it will achieve a steady status. Numerous clinical studies of OCT show a mean symptomatic response rate of $73 \%$ (range, $50 \%-100 \%)$ in GEP-NETs [11]. OCT and OCT LAR has similar mean 
biochemical response (partial plus complete responses) rate of $51 \%$ but with varied ranges (OCT, $28 \%-77 \%$; OCT LAR, 31.5\%-100\%) [12].

As yet, however, there is few report on application of SST analogues in advanced NECs of the paranasal sinuses. As IHC analysis had identified the overexpression of all SSTRs subtypes (SSTRs 1-5) of tumor cells in present case, we consider somatostatin analog (SSA) a potential therapy regimen for this patient.

In our case, the patient was treated with daily OCT for 2 weeks followed by monthly OCT LAR and achieved a successful control of symptoms and improved quality of life (QOL). A significant reduction in both tumor size and enhancement of tumor were confirmed by contrast MRI. During the 89 months of follow-up, the patient well responded to OCT/OCT LAR treatment, and remained clinically well with increased EQ-5D scores.

Numerous studies have shown OCT and OCT LAR are generally well tolerated [13]. In this case, only mild local reactions at the injection site were found when OCT/OCT LAR therapy was initiated. No serious drug-related adverse effects, according to the definition of NCI-CTC version 3.0, were observed on the blood, liver, kidneys, or heart in this patient.

\section{Conclusion}

In conclusion, our case report demonstrated that OCT/OCT LAR induces a sustained regression of sphenoidal NEC and is well tolerated by the patient. It may be a reasonable treatment option for advanced NECs in paranasal sinuses and is worth further investigation and research on it.

\section{Acknowledgment}

This work was supported by National Science Foundation of Jiangsu Province (BK20141492), Jiangsu 333 project (BRA2016517) and Priority Academic Program Development of Jiangsu Higher Education Institutions.

\section{References}

1. Mitchell EH, Diaz A, Yilmaz T, Roberts D, Levine N, et al. (2012) Multimodality treatment for sinonasal neuroendocrine carcinoma. Head Neck 34: 1372-1376.
2. Waldron J, Witterick I (2003) Paranasal sinus cancer: caveats and controversies. World J Surg 27: 849-855.

3. Giovacchini G, Nicolas G, Forrer F (2012) Peptide receptor radionuclide therapy with somatostatin analogues in neuroendocrine tumors. Anticancer Agents Med Chem 12: 526-542.

4. Durán-Prado M, Malagón MM, Gracia-Navarro F, Castaño JP (2008) Dimerization of $G$ protein-coupled receptors: new avenues for somatostatin receptor signalling, control and functioning. Mol Cell Endocrinol. 286: 63-68.

5. Parry JJ, Chen R, Andrews R, Lears KA, Rogers BE (2012) Identification of critical residues involved in ligand binding and $\mathrm{G}$ protein signaling in human somatostatin receptor subtype 2. Endocrinology 153: 2747-2755.

6. Bousquet C, Lasfargues C, Chalabi M, Billah SM, Susini C, et al. (2012) Clinical review: Current scientific rationale for the use of somatostatin analogs and mTOR inhibitors in neuroendocrine tumor therapy. J Clin Endocrinol Metab 97: 727-737.

7. Pyronnet S, Bousquet C, Najib S, Azar R, Laklai H, et al. (2008) Antitumor effects of somatostatin. Mol Cell Endocrinol 286: 230-237.

8. Reubi JC, Waser B, Cescato R, Gloor B, Stettler C, et al. (2010) Internalized somatostatin receptor subtype 2 in neuroendocrine tumors of octreotide-treated patients. J Clin Endocrinol Metab 95: 2343-2350.

9. Csaba Z, Peineau S, Dournaud P (2012) Molecular mechanisms of somatostatin receptor trafficking. J Mol Endocrinol 48: R1-12.

10. Astruc B, Marbach P, Bouterfa H, Denot C, Safari M, et al. (2005) Longacting octreotide and prolonged-release lanreotide formulations have different pharmacokinetic profiles. J Clin Pharmacol 45: 836-844.

11. Oberg K, Kvols L, Caplin M, Delle Fave G, de Herder W, et al. (2004) Consensus report on the use of somatostatin analogs for the management of neuroendocrine tumors of the gastroenteropancreatic system. Ann Oncol 15: 966-973.

12. Modlin IM, Pavel M, Kidd M, Gustafsson BI (2010) Review article: somatostatin analogues in the treatment of gastroenteropancreatic neuroendocrine (carcinoid) tumours. Aliment Pharmacol Ther 31: 169-188.

13. Rinke A, Müller HH, Schade-Brittinger C, Klose KJ, Barth P, et al. (2009) Placebo-controlled, double-blind, prospective, randomized study on the effect of octreotide LAR in the control of tumor growth in patients with metastatic neuroendocrine midgut tumors: a report from the PROMID Study Group. J Clin Oncol 27: 4656-4663. 